\title{
Diffusion-weighted perinatal postmortem magnetic resonance imaging as a marker of postmortem interval
}

\author{
Owen J. Arthurs • Gemma C. Price • David W. \\ Carmichael • Rod Jones • Wendy Norman • \\ Andrew M. Taylor • Neil J. Sebire
}

Received: 9 July 2014 /Revised: 15 September 2014 / Accepted: 19 November 2014 / Published online: 18 December 2014

(C) The Author(s) 2014. This article is published with open access at Springerlink.com

\begin{abstract}
Objective To evaluate perinatal body organ apparent diffusion coefficient (ADC) values at postmortem magnetic resonance imaging (PMMR) in order to evaluate postmortem changes. Methods Postmortem diffusion-weighted imaging (DWI) of the thorax and abdomen were performed with diffusion gradient values $b=0,500$, and $1000 \mathrm{~s} / \mathrm{mm}^{2}$ on 15 foetal and childhood cases (mean $33.3 \pm 7.8$ weeks gestation) compared to 44 live infants (mean age $75.5 \pm 53.4$ days). Mean ADC values were calculated from regions of interest (ROIs) for the lungs, liver, spleen and renal cortex, compared to normative live infantile body ADC values of similar gestational age. Results Mean ADC values were significantly lower in postmortem cases than in normal controls for liver $\left(0.8810^{-3} \mathrm{~mm}^{2} /\right.$ $\mathrm{s} \pm$ SD 0.39 vs. $1.13 \pm 0.13 ; \mathrm{p}<0.05)$ and renal cortex $(0.85 \pm$ 0.26 vs. $1.19 \pm 0.13 ; \mathrm{p}<0.05)$ but not spleen or muscle. Mean lung ADC values were significantly higher than normal
\end{abstract}

O. J. Arthurs $(\bowtie) \cdot$ G. C. Price

Department of Radiology, Great Ormond Street Hospital for Children

NHS Foundation Trust, London WC1N 3JH, UK

e-mail: owen.arthurs@gosh.nhs.uk

O. J. Arthurs $\cdot$ D. W. Carmichael

Imaging and Biophysics, UCL Institute of Child Health, London, UK

R. Jones $\cdot$ W. Norman $\cdot$ A. M. Taylor

Cardiorespiratory Division, Great Ormond Street Hospital for

Children NHS Foundation Trust, London, UK

R. Jones $\cdot$ W. Norman $\cdot$ A. M. Taylor

Centre for Cardiovascular Imaging, UCL Institute of Cardiovascular

Science, London, UK

\section{N. J. Sebire}

Department of Histopathology, Great Ormond Street Hospital for Children NHS Foundation Trust, London, UK

\section{N. J. Sebire}

Histopathology, UCL Institute of Child Health, London, UK controls $(1.06 \pm 0.18$ vs. $0 \pm 0 ; p<0.001)$, and there was a significant correlation between postmortem interval and lung $\operatorname{ADC}\left(\mathrm{R}^{2}=0.55\right)$.

Conclusion Lung PMMR ADC values are related to postmortem interval, making them a potential marker of time since death. Further research is needed to understand the organspecific changes which occur in the postmortem period.

Key Points

- Liver and spleen PMADC values were lower than controls.

- Lung ADC changes correlate with PM interval.

- These findings may be useful in medicolegal cases.

Keywords Autopsy $\cdot$ Postmortem $\cdot$ MRI $\cdot$ Diffusion · Paediatric $\cdot$ Perinatal
Abbreviations
ADC Apparent diffusion coefficient
DTI Diffusion tensor (magnetic resonance) imaging
DWI Diffusion weighted (magnetic resonance) imaging
(PM)MR (postmortem) magnetic resonance imaging

\section{Introduction}

Postmortem imaging now plays a significant role in the paediatric and perinatal minimally invasive autopsy, largely due to a decline in parental acceptance of traditional autopsy techniques fuelling a drive to develop a novel less invasive postmortem approach $[1,2]$. Several different imaging modalities are being employed in this regard, including postmortem (PM) skeletal radiographs, typically used to diagnose skeletal dysplasias and assess bone gestational changes [3], and cross-sectional imaging techniques including PM computerized tomography $(\mathrm{CT})$ and $\mathrm{PM}$ magnetic resonance 
imaging (PMMR), which have been reviewed extensively elsewhere [4]. Postmortem CT is rapidly available, and readily gives vascular and bone detail, it has not been shown to be diagnostically accurate for soft tissue abnormalities in fetuses and children. PMMR has been shown to have high diagnostic accuracy for perinatal abnormalities, in both small preliminary (e.g., Breeze et al. [5]) and large blinded studies [6], and PMMR is widely believed to likely become the mainstay of a less invasive perinatal imaging service [4].

However, there remain areas of potential diagnostic improvement, with true ischaemia and necrosis difficult to evaluate with conventional magnetic resonance (MR) techniques [7]. Diffusion-weighted imaging (DWI) is a structural MR imaging technique which measures the diffusivity of water molecules. It can be measured quantitatively and expressed as an apparent diffusion coefficient (ADC) value, such that a lower ADC value represents a tissue with more restricted water diffusivity. ADC measures of body organs have been used to evaluate tumour characteristics in paediatric tumours, such as Wilms tumours and neuroblastoma [8-10].

Following death, there are several changes in tissues due to tissue breakdown (autolysis) and decomposition. In the initial hours following death, tissue ischaemia will occur, followed by a period of cell lysis, membrane breakdown, fluid redistribution and gas formation/putrefaction [11]. We hypothesise that PMMR DWI changes in individual organs will correspond to these changes, with an initial decrease in ADC values secondary to ischaemia (such as in stroke), followed by a possible increase in ADC values following cell breakdown and autolysis. Human PM specimens have recently been used to confirm DWI tractography [12], and several animal studies have suggested an effect of PM interval on DWI changes in the brain [13-17].

This study was therefore designed to establish whether (a) DWI changes are detectable by PMMR in fetuses and stillbirths, (b) PMMR DWI changes increase following death, possibly due to cellular barriers breaking down and increasing water movement, and (c) PMMR DWI changes correlate with PM interval, and could be possible surrogate markers for autolysis.

\section{Methods}

Study cohort

Live infants We retrospectively searched our hospital database for all cases of thoraco-abdominal MRI in infants under the age of 6 months of age over a 5-year period (April 2008 to July 2013). All live patients/guardians gave consent for MR imaging including DWI as part of their clinical care. We excluded cases in which DWI was not performed in the body organs, or was of inadequate quality for assessment, or those in whom there was significant pathological involvement of the abdominal organs, such as by solid tumours, metastases, or vascular malformations. Age and gender were acquired from clinical attendance.

Postmortem cases We prospectively collected DWI sequences on all PM foetal and stillbirth cases referred to our institution in the time period January-October 2013. We excluded cases in which DWI was of inadequate quality, incomplete datasets, and those in whom there was pathological involvement of the abdominal organs. Written informed consent was obtained for all patients for clinical pre-autopsy PMMR as part of our institution's clinical PM assessment. Bodies were stored in a mortuary at $4{ }^{\circ} \mathrm{C}$ and PMMR was performed out of hours, causing least disturbance to clinical services. Demographic data acquired from the clinical notes included age (gestation in weeks), gender, intra-uterine interval (number of days of intra-uterine retention if stillborn or termination of pregnancy), maceration score (visual index assessed by pathologist at autopsy: 0 none, 1 mild, 2 moderate/ severe), and PM interval (days from death to imaging).

\section{Magnetic Resonance Imaging}

All MR imaging was performed at $1.5 \mathrm{~T}$ (Avanto, Siemens Medical Solutions, Erlangen, Germany), with a conventional phased array body coil. MR imaging in live cases included a whole-body $3 \mathrm{D}_{2}$-weighted turbo spin echo (TSE, TR $3500 \mathrm{~ms}$, TE $276 \mathrm{~ms}$, voxel size $0.8 \times 0.8 \times 0.8 \mathrm{~mm}$, two averages, variable acquisition time depending upon respiratory gating) and PM body imaging included a 3D CISS (Constructive Interference Steady State sequence, a modified gradient-echo balanced steady-state free precession sequence with T2/T1 contrast; TR/TE 9.1/4.5 ms, NEX 8, flip angle $70^{\circ}$, voxel sizes $0.8 \times 0.8 \times 0.8 \mathrm{~mm}$; acquisition time $4.2 \mathrm{mi}-$ nutes) for clinical diagnostic purposes and identification of body organs [18].

DWI was performed using single-shot spin-echoplanar imaging (EPI) in the axial plane, with the following parameters: 19 slices in three non-collinear axis directions, EPI factor 95, TR $2700 \mathrm{~ms}$; TE $96 \mathrm{~ms}$; FOV $230 \mathrm{~mm} ; 128 \times 128$ matrix, 5-mm slice thickness with 1-mm gap, acquisition time 90 seconds. Diffusion gradient values were $b=0,500$, and $1000 \mathrm{~s} / \mathrm{mm}^{2}$ for all cases (including PMMR), to ensure consistency between measurements.

Diffusion-weighted imaging analysis

From the native DWI acquisition, ADC maps were obtained on the acquisition console (Syngo, Siemens Medical Solutions, Erlangen, Germany). Data were then transferred to Osirix (open source code; http://www.osirix-viewer.com) for 
ADC measurements in different regions of interest (ROI). For each study, five circular regions of interest (ROIs) were drawn manually and plotted on ADC maps by the same operator, a radiology Intern with 2 years radiology experience (GCP). These were placed on the (1) liver parenchyma, (2) spleen, (3) left renal cortex (4) left psoas muscle (psoas used as a control value), and (5) lung parenchyma. For each ROI, a mean ADC value $\left(10^{-3} \mathrm{~mm}^{2} / \mathrm{s}\right) \pm$ standard deviation (SD) was obtained. The centre of the ROI was placed on the organ of interest, with as large a circular ROI as possible while avoiding adjacent structures (such as major blood vessels in the liver), but with relative $\mathrm{SD}<10 \%$ of the mean, to ensure homogeneity - typically $25 \mathrm{~mm}^{2}$. A random selection of 12 images were also assessed by a radiology consultant with 7 years radiology experience (OJA), giving a measure of inter-observer reproducibility. We did not measure intra-observer reproducibility.

\section{Temperature correction}

As water diffusion is dependent on temperature, and PM examinations were performed on refrigerated bodies, a temperature correction was applied to PM ADC values. This was performed according to Kozak et al. [19], who give the relationship between temperature and diffusion in unrestricted water as

$$
T=\frac{2256.74 K}{\ln \left[\frac{4392.21 \times 10^{-3} \mathrm{~mm}^{2} / \mathrm{s}}{\mathrm{Dmm}^{2} / \mathrm{s}}\right]}-273.15 K
$$

Where $\mathrm{T}$ is temperature in units of Celsius, $\mathrm{D}$ is the diffusion in $\mathrm{mm}^{2} / \mathrm{s}$.

Therefore rearranging for $\mathrm{D}$ gives

$$
D=\frac{4392.21 \times 10^{-3}}{\exp \left(\frac{2256.74}{T+273.15}\right)}
$$

We assumed body temperatures of PM and live cases to be $4^{\circ} \mathrm{C}$ and $37^{\circ} \mathrm{C}$, respectively, giving a correction of 0.0030 / $0.0013=2.38$.

\section{Statistical analysis}

Quantitative values are given as mean ROI ADC values $\left(10^{-3} \mathrm{~mm}^{2} / \mathrm{s}\right) \pm \mathrm{SD}$. ADC values were plotted against age (gestation), intra-uterine interval, maceration score, and PM interval for PM cases, and age at the time of scanning in live patients. We also compared live infant ADC values to those quoted in the literature [10]. Statistical analysis was performed using Student's t-test and Fisher's exact test at the $5 \%$ level of significance level, using SPSS 19.0 for Windows (IBM UK Ltd, Portsmouth, UK). For variability, we calculated bias between observers (mean difference) and $95 \%$ limits of agreement.

\section{Results}

Live infant ADC

We identified 44 complete DWI datasets in infants $<6$ months of age who had undergone thoraco-abdominal imaging at our institution, which included 31 males and 13 females (e.g. Fig. 1). Mean age was $75.5 \pm 53.4$ days, with range $1-$ 178 days of age (Table 1). The majority underwent followup imaging for solid tumours, which did not involve the abdominal viscera.

Mean ADC values $\left(10^{-3} \mathrm{~mm}^{2} / \mathrm{s}\right) \pm$ standard deviation (SD) for individual abdominal organs were significantly lower than quoted values in the literature: liver $1.13 \pm 0.14$ (range in literature $1.4-1.8$ ); spleen $0.79 \pm 0.09$ (range in literature $0.7-1.2$ ); and renal cortex $1.19 \pm 0.13$ (range in literature $1.8-2.2$; Table 1 ). There was no returnable ADC signal from lung parenchyma in any live case (mean $0 \pm 0)$. There was no effect of age on ADC values $\left(\mathrm{R}^{2}<0.1\right.$ for all organs).

Inter-observer variability (bias $\pm 95 \%$ limits of agreement) of 12 randomly selected cases was $0.04 \pm 0.06$ for liver, $0.03 \pm$ 0.04 for spleen, and $0.03 \pm 0.04$ for renal cortex, the equivalent of $2.7-4.3 \%$ of the variability of mean ADC.

\section{Postmortem ADC}

We identified 15 fetal and neonatal PMMR DWI cases (mean 33.3 \pm 7.8 weeks gestation, range 23-50) including six males and nine females (Table 2; e.g. Fig. 2). Temperature corrected mean ADC values $\left(\times 10^{-3} \mathrm{~mm}^{2} / \mathrm{s}\right)$ were significantly lower in $\mathrm{PM}$ cases than in normal controls in the liver $(0.88 \pm \mathrm{SD} 0.39$ vs. $1.13 \pm 0.13, \mathrm{p}<$ $0.005)$ and kidney $(0.85 \pm 0.26$ vs. $1.19 \pm 0.13, \mathrm{p}<0.005)$, but did not reach significance for the spleen $(0.67 \pm 0.25$ vs. $0.79 \pm 0.09, \mathrm{p}=0.132)$ or muscle $(1.26 \pm 0.40$ vs. $1.11 \pm$ $0.12, p=0.08$; Table 1; Fig. 3). The normal liver/spleen (PM 1.31, live 1.44) and spleen/kidney ratios (PM 1; live 0.96) were maintained. We excluded two cases who had abnormal cystic kidneys from the renal ADC analysis only.

Mean ADC values of the lungs were significantly higher than in normal controls $(1.06 \pm 0.18$ vs. $0 \pm 0, \mathrm{p}<0.001$; Table 1). There was a significant correlation between mean 
Fig. 1 Infant thoraco-abdominal diffusion-weighted imaging (DWI). Axial T2-weighted magnetic resonance (MR) imaging (left; a - c) and apparent diffusion coefficient (ADC) maps from DWI sequences (right; $d$ - $f$ ) in a 2-month-old boy who underwent MR imaging for a congenital sacral abnormality (not shown). Axial slices illustrate the lungs (a), liver and spleen (b) and kidneys (c), with corresponding regions of interest drawn on $\mathrm{ADC}$ maps over the lungs (d), liver and spleen (e), renal cortex (f) and muscle (not shown). No appreciable ADC signal was detected from the lungs (d)

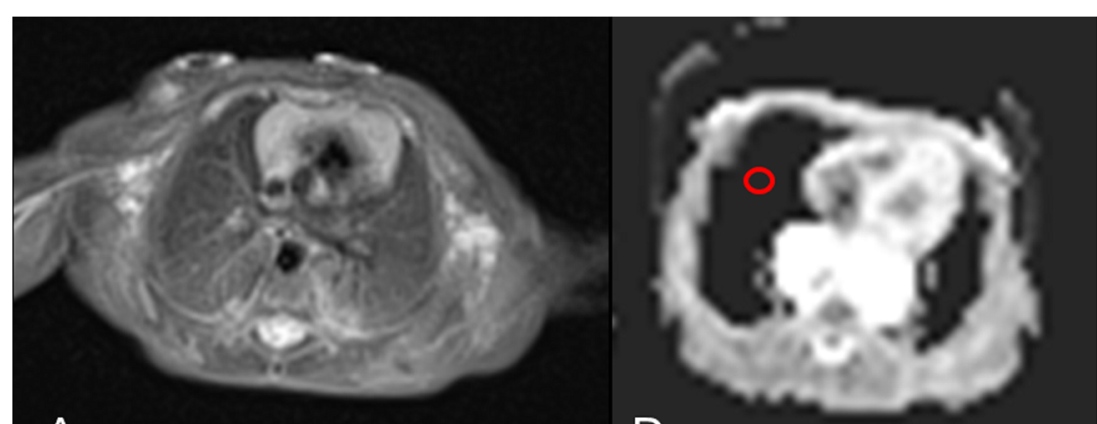

A

D

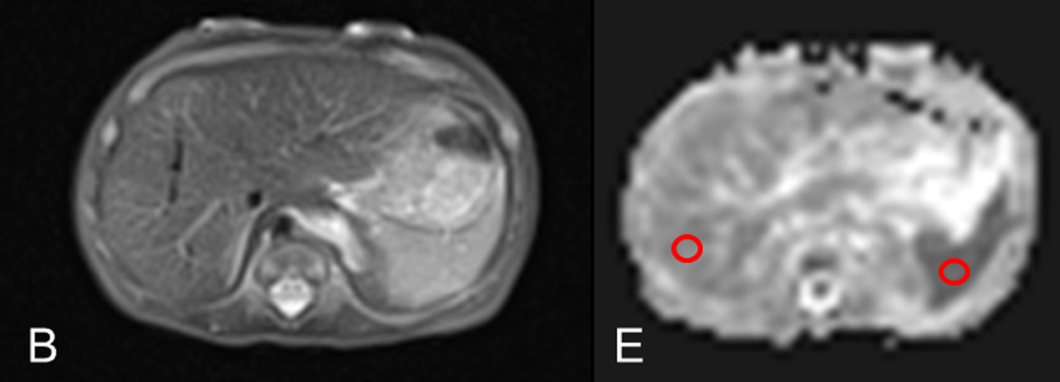

B

C

F
ADC values and PM interval for lung parenchyma (non-linear correlation $\mathrm{y}=0.28 \ln (\mathrm{x})+0.44 ; \mathrm{R}^{2}=0.66$; Fig. 4)., but not for gestational age, intra-uterine retention interval nor maceration score. There were no correlations between ADC values of any abdominal organ and any of these parameters $\left(\mathrm{R}^{2}<0.1\right.$ for all organs).
Table 1 Demographics and apparent diffusion coefficient (ADC) values of live and postmortem (PM) cases by body organ

$* *=\mathrm{p}<0.05$

\begin{tabular}{lll}
\hline & Live infants & PM cases \\
\hline Number (n) & 44 & 15 \\
Male : Female & $30: 14$ & $6: 9$ \\
Mean age & $75.5 \pm 53.4$ days & $33.3 \pm 7.8$ wks \\
Age range & $1-178$ days & $23-50 \mathrm{wks}$ \\
Lung ADC $\left(10^{-3} \mathrm{~mm}^{2} / \mathrm{s}\right)$ & $0 \pm 0$ & $1.06 \pm 0.18 * *$ \\
Liver ADC $\left(10^{-3} \mathrm{~mm}^{2} / \mathrm{s}\right)$ & $1.13 \pm 0.13$ & $0.88 \pm 0.39 * *$ \\
Spleen ADC $\left(10^{-3} \mathrm{~mm}^{2} / \mathrm{s}\right)$ & $0.79 \pm 0.09$ & $0.69 \pm 0.25$ \\
Renal cortex ADC $\left(10^{-3} \mathrm{~mm}^{2} / \mathrm{s}\right)$ & $1.19 \pm 0.13$ & $0.85 \pm 0.26 * *$ \\
Muscle $\left(10^{-3} \mathrm{~mm}^{2} / \mathrm{s}\right)$ & $1.11 \pm 0.12$ & $1.26 \pm 0.40$ \\
\hline
\end{tabular}


Table 2 Details of 15 cases fetal and neonatal PMMR cases, including demographic details as well as post mortem maceration score and interval. Cause of death was obtained from autopsy

\begin{tabular}{|c|c|c|c|c|c|c|c|}
\hline Case & $\begin{array}{l}\text { Foetal }(\mathrm{F}) \\
\text { or neonatal } \\
(\mathrm{N})\end{array}$ & Gender & $\begin{array}{l}\text { Gestational } \\
\text { Age (wks) }\end{array}$ & $\begin{array}{l}\text { Intra-uterine } \\
\text { retention time } \\
\text { (days) }\end{array}$ & $\begin{array}{l}\text { Maceration score } \\
(0 \text { none, } 1 \text { mild, } 2 \\
\text { mod })\end{array}$ & $\begin{array}{l}\text { Postmortem } \\
\text { interval (days) }\end{array}$ & Cause of death or diagnosis \\
\hline 1 & $\mathrm{~F}$ & Female & 36 & 2 & 2 & 9 & Brain malformation - intracranial haemorrhage \\
\hline 2 & $\mathrm{~F}$ & Female & 26 & 1 & 0 & 7 & $\begin{array}{l}\text { Severe intrauterine growth restriction } \\
\text { and placental abruption }\end{array}$ \\
\hline 3 & $\mathrm{~F}$ & Female & 23 & 0 & 0 & 10 & Brain malformation - callosal agenesis \\
\hline 4 & $\mathrm{~F}$ & Female & 32 & 2 & 1 & 8 & Neural tube defect \\
\hline 5 & $\mathrm{~N}$ & Male & 50 & 0 & 0 & 2 & $\begin{array}{l}\text { Unexplained sudden unexplained infant death } \\
\text { (cosleeping) }\end{array}$ \\
\hline 6 & $\mathrm{~F}$ & Male & 32 & 1 & 0 & 9 & $\begin{array}{l}\text { Severe uteroplacental disease and intracranial } \\
\text { haemorrhage }\end{array}$ \\
\hline 7 & $\mathrm{~F}$ & Female & 32 & 0 & 0 & 15 & Brain malformation - cortical malformation \\
\hline 8 & $\mathrm{~F}$ & Male & 38 & 1 & 0 & 22 & $\begin{array}{l}\text { Unexplained intrauterine death - } \\
\text { obstetric cholestasis }\end{array}$ \\
\hline 9 & $\mathrm{~F}$ & Male & 38 & 0 & 0 & 11 & Intrapartum death \\
\hline 10 & $\mathrm{~F}$ & Male & 41 & 0 & 0 & 10 & Intrapartum death \\
\hline 11 & $\mathrm{~F}$ & Female & 23 & 1 & 0 & 18 & Congenital heart disease (aortic coarctation) \\
\hline 12 & $\mathrm{~F}$ & Male & 32 & 0.5 & 1 & 8 & Renal dysplasia \\
\hline 13 & $\mathrm{~F}$ & Female & 41 & 0.5 & 0 & 8 & Unexplained intrauterine death \\
\hline 14 & $\mathrm{~N}$ & Female & 22 & 0.5 & 0 & 13 & Neural tube defect \\
\hline 15 & $\mathrm{~F}$ & Female & 33 & 1 & 2 & 11 & Renal dysplasia \\
\hline Mean & & & $33.3 \pm 7.8 \mathrm{wk}$ & & & $10.7 \pm 4.8$ days & \\
\hline
\end{tabular}

\section{Discussion}

The findings of this study demonstrate that DWI characteristics of thoraco-abdominal organs change following death, and can be evaluated using PMMR. Liver and renal cortex ADC values were lower in PM cases than normal controls. Lung $\mathrm{ADC}$ values were higher, and there was a significant correlation between lung ADC and PM interval (but not with ADC of any abdominal organ). Lung ADC changes could therefore be useful in estimating PM interval in future cases, although further research is needed to understand the fall in organ ADC which occurs during the PM period.

Our first observation was that live infant $\mathrm{ADC}$ values in our study were significantly lower than values quoted in the literature, for all of the abdominal solid organs (liver, spleen and renal cortex). This is likely to reflect both variation between acquisition protocols between different machines and vendors, as well as a possible correlation between ADC and age. Gawande et al. [10] present a summary table of common values in the literature, but most of the studies quoted were in adults. It is recognised that paediatric solid organ ADC values are typically lower than those in adults [20], perhaps due to changes in anatomy in growing tissues, although we were unable to demonstrate this amongst the live children in our study. The age-dependence correlation in live infants may be much shallower in gradient (over years, rather than weeks or months) than we could detect in our population under 6 months.

Our second observation was that there were differences between live and PM ADC values in different solid organs, particularly the liver and renal cortex. This may be related to inherent water content of different organs and their rate of decomposition. Although the results did not reach statistical significance for the spleen, the same trend as seen for the liver and kidney was observed, and so the same process is likely to occur. Muscle was used as a control and no trend was observed for live versus PM differences. As the normal ratio of values between organs was retained, we surmise that the same changes are therefore being seen in most organs, in keeping with generalised rather than abdominal organ specific changes. What these changes are on a cellular basis remains to be determined.

Our third observation from this dataset was that lung ADC changes correlate with postmortem interval in this study. As lung $\mathrm{ADC}$ values were higher, and body organ $\mathrm{ADC}$ values were lower than normal controls, we hypothesise that two different processes account for the ADC changes observed in this study. Lung ADC changes are likely to represent fluid accumulation, since there is no signal obtained from lung parenchyma in live infants as the lung volume is predominantly composed of air. Accumulation of fluid in the lungs and pleural spaces over time is a recognised phenomenon following death, but the imaging correlates of this have not 
Fig. 2 Thoraco-abdominal postmortem magnetic resonance (PMMR) diffusion-weighted imaging (DWI). Axial T2weighted MR imaging (left; a - c) and apparent diffusion coefficient (ADC) maps from DWI sequences (right; $\mathrm{d}$ - $\mathrm{f}$ ) in a 36week gestation foetus that underwent PMMR for a congenital brain malformation (not shown). Axial slices illustrate the lungs (a), liver and spleen (b) and kidneys (c), with corresponding regions of interest drawn on ADC maps over the lungs (d), liver and spleen (e), renal cortex (f) and muscle (not shown)

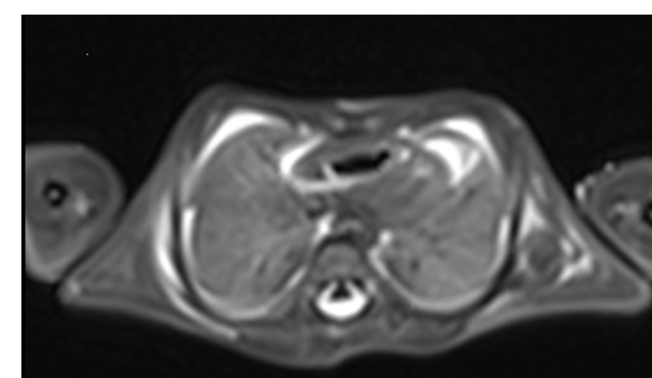

A D

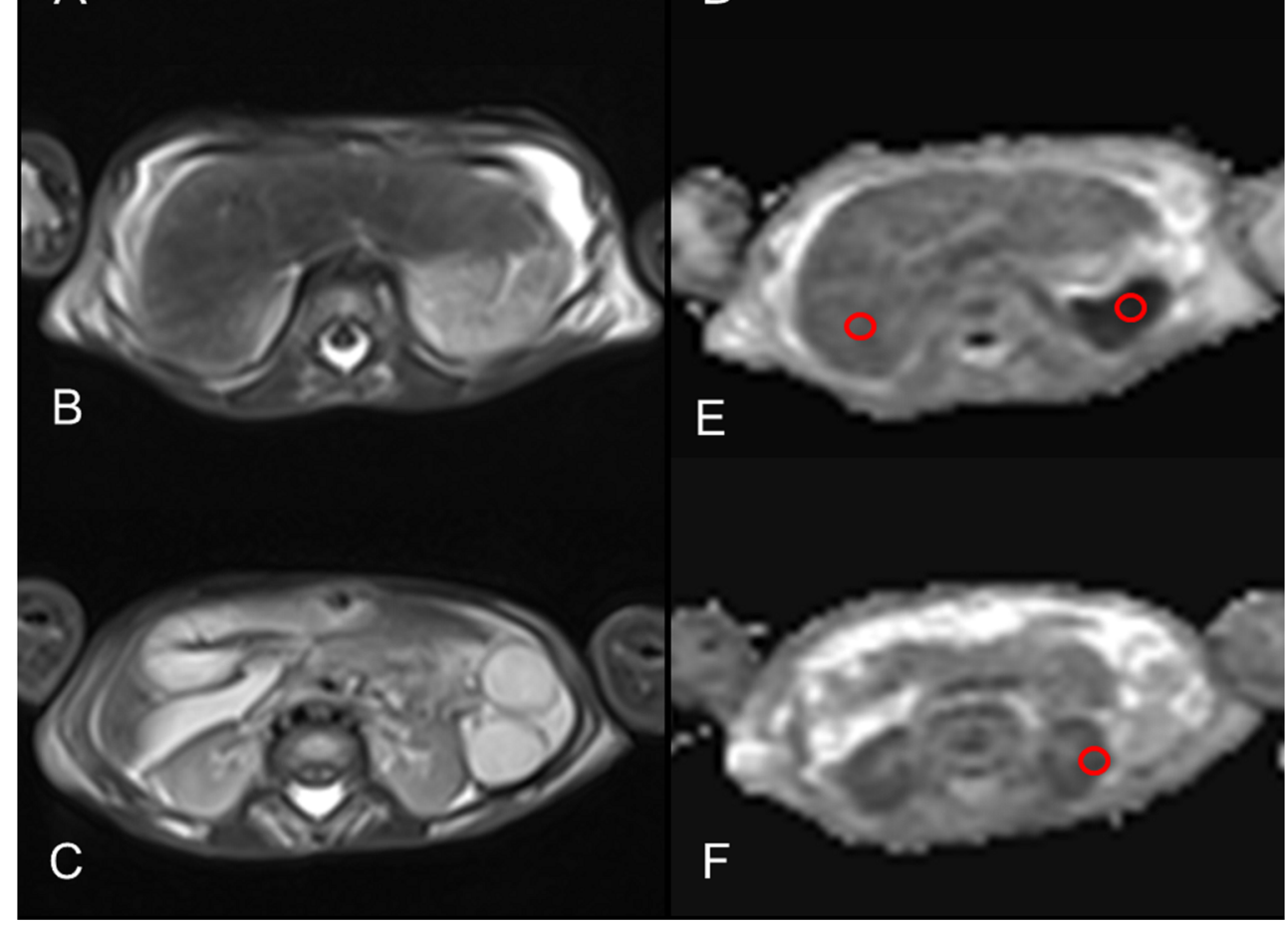

previously been identified, nor quantified to correlate with PM interval. The accuracy of PMMR in determining PM interval

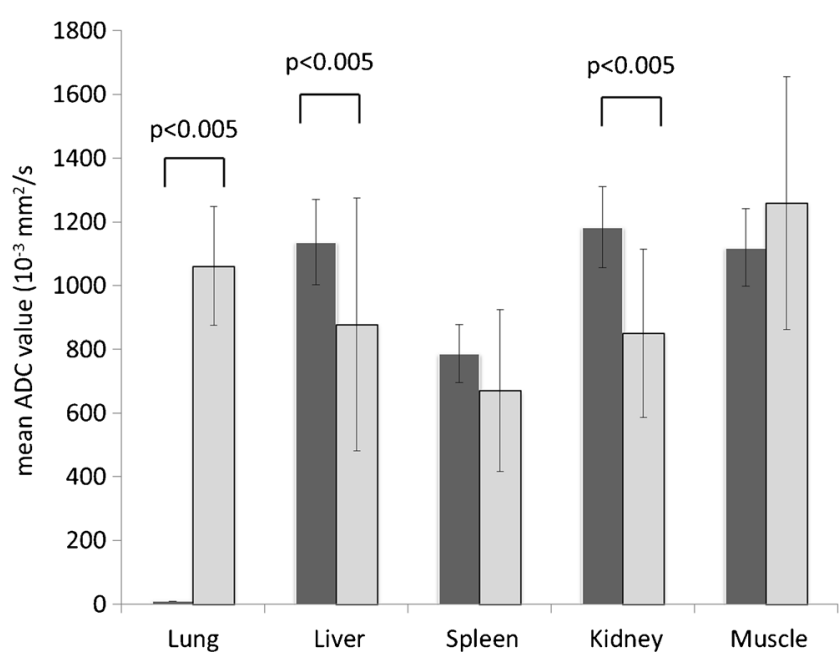

Fig. 3 Comparison of live vs post-mortem magnetic resonance (PMMR) apparent diffusion coefficient (ADC) values. Mean ADC values for live infant and PMMR thoracic and abdominal organs \pm standard deviation for each organ may be useful in cases in which the time interval since death is unknown, such as delayed presentation and forensic cases.

Our final observation was that there was also no correlation between PM interval and ADC changes in other body organs in our population. Both the timing and nature of these changes are interesting. Our results imply a rapid change in ADC within hours after death which stabilises within 24-72 hours, which we did not detect given our PM interval range of days rather than hours after death. Several previous studies have described an effect of PM interval on DWI or DTI characteristics of animal brains in the first few hours after death [13, 14], although different immersion/ fixation techniques may have affected these results [13-16]. The present study avoids the interaction of immersion and fixation techniques by imaging whole-body PM cases. Other studies have shown that ADC values in the PM adult brain are significantly lower than normal controls (49-72 \% lower), and may correlate with PM interval, although only three time intervals were compared ( $<24$ hours, $24-48$ hours, and $>48$ hours) [17]. Interestingly, our study found a correlation with lung ADC which continued 
Fig. 4 Lung apparent diffusion coefficient (ADC) changes with postmortem (PM) interval. Lung ADC changes correlate with PM interval $\left(\mathrm{y}=0.28 \ln (\mathrm{x})+0.44 ; \mathrm{R}^{2}=\right.$ 0.66 )

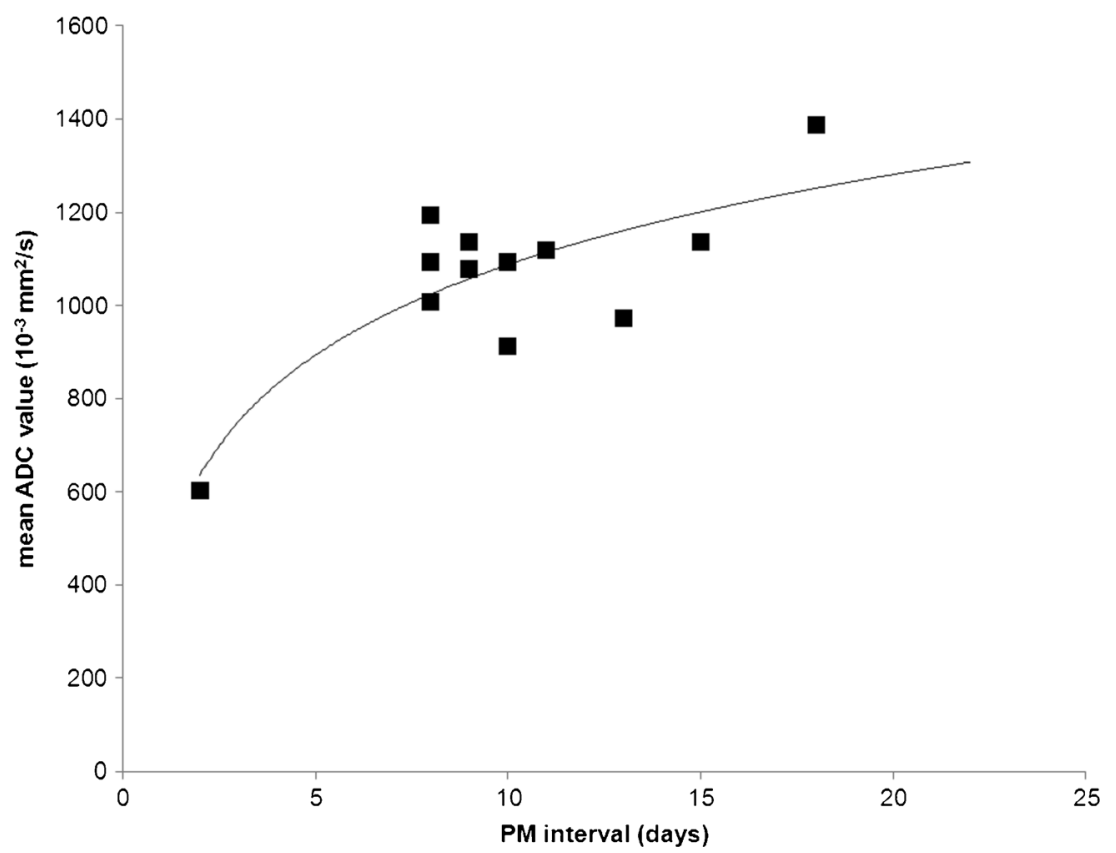

beyond 14 days. However, the degree of inter-individual variability (between cases) for organ ADC values may be greater than the correlation with PM interval, thus limiting the accuracy of results. Serial measurements on the same case may provide further data regarding this mechanism.

We acknowledge that unlike lung changes, other organ ADC changes are unlikely to represent simple fluid accumulation. DWI measures the diffusivity, or freedom of movement of water molecules, with the magnitude of signal loss between dephasing and rephasing gradients proportional to the diffusivity of the tissue [21]. Several factors interact to change the ADC values, including water content, tissue cellularity and integrity of intracellular membranes [22]. Whilst there was a decrease in ADC values likely secondary to ischaemia, histological analysis is required to shed light on which of these factors is likely to contribute greatest to the imaging changes identified. Autolysis-associated histological changes are well recognised in PM samples, with variation between tissue types, but quantitation of such changes in relation to factors including cause of death and PM interval is difficult. The liver and kidney are both metabolically and enzymatically active tissues and it is generally accepted that autolytic activity causes cellular breakdown, but the precise mechanism relating changes in ADC values over time to histological features would require serial sampling from the early and immediate PM period, perhaps in an animal study.

Despite this being the first study which addresses changes in foetal and stillbirth organs using PMMR, the limitations include the relatively small sample size, and that we were unable to further elucidate specific mechanisms of body organ changes across different organ types. The data is also based on a comparison of foetal PM cases and live infants, with a small (gestational) age difference. An alternative dataset for comparison with foetal PM ADC values would be antenatal foetal body organ and lung MRI, but these were not available at the time of this study. However, results from the foetal MRI literature largely support our findings. For example, Savelli et al. [23] found that normal foetal renal ADC values ranged between 1.06 and $1.33 \times 10^{-3} \mathrm{~mm}^{2} / \mathrm{s}$, which supports our findings as these values are comparable to our infant group but significantly higher than the PM group. Other groups have assessed foetal lung ADC changes with debate as to whether there is true a correlation with gestational age. Manganaro et al. [24] found that foetal lung values increased from $1.2 \times 10^{-3} \mathrm{~mm}^{2} / \mathrm{s}$ at 18 weeks to $3.9 \times 10^{-3} \mathrm{~mm}^{2} / \mathrm{s}$ at 36 weeks, with a mean lung ADC of $2.35 \pm 0.6 \times 10^{-3} \mathrm{~mm}^{2} / \mathrm{s}$, and correlation suggesting that foetal lung ADC can be used as a surrogate marker of lung maturity. However, two other papers document foetal lung ADCs between 1.63 and $2.13 \times 10^{-3} \mathrm{~mm}^{2} / \mathrm{s}$ which were highly reproducible and largely dependent upon gravitational changes on the lung irrespective of gestational age $[25,26]$. Our PM lung ADC values in a similar group of late foetal deaths are much lower than the values quoted for normal foetuses, and we did not find a correlation with gestation.

We also used a temperature correction based on unrestricted diffusion, whereas different properties in different tissues could display different sensitivity to temperature. Whilst this could bias the between-group comparison (in favour of not finding a difference between groups), it does not affect the observed correlation between PM interval and lung ADCs. In addition, this method has been used to measure temperature changes in tissue with reasonable accuracy [19]. Finally, we excluded cases with lung abnormalities or organ disease, in 
which the presence of additional pathology could affect these results.

In summary, PM lung ADC values probably reflect fluid accumulation in the lungs after death, and correlate with PM interval. This could be a useful imaging marker of PM interval in future cases. However, further research is needed to understand the timing and nature of the different changes in individual organ $\mathrm{ADC}$ values which occurs soon after death.

Acknowledgements The scientific guarantor of this publication is Owen J. Arthurs. The authors of this manuscript declare no relationships with any companies, whose products or services may be related to the subject matter of the article.

OA is funded by an NIHR Clinician Scientist Fellowship, AMT and NJS are funded by NIHR Senior Investigator awards, Great Ormond Street Children's Charity and the Great Ormond Street Hospital Biomedical Research Centre. None of the funding bodies had any role in analysis of data, results or conclusions of the study.

This article presents independent research funded by the National Institute for Health Research (NIHR) and supported by the Great Ormond Street Hospital Biomedical Research Centre. The views expressed are those of the author(s) and not necessarily those of the National Health Service (NHS), the NIHR or the Department of Health.

One of the authors has significant statistical expertise. Written informed consent was obtained from all subjects (patients) in this study. None of the study has been previously reported. Methodology: prospective recruitment with retrospective comparison, case-control study.

Open Access This article is distributed under the terms of the Creative Commons Attribution Noncommercial License which permits any noncommercial use, distribution, and reproduction in any medium, provided the original author(s) and the source are credited.

\section{REFERENCES}

1. Whitby EH, Paley MN, Cohen M, Griffiths PD (2006) Post-mortem fetal MRI: what do we learn from it? Eur J Radiol 57:250-255

2. Arthurs OJ, Taylor AM, Sebire NJ (2013) The less invasive perinatal autopsy: current status and future directions. Fetal Matern Med Rev 24:45-59

3. Calder AD, Offiah AC (2014) Fetal radiography for suspected skeletal dysplasia : technique, normal appearances and diagnostic approach. Pediatr Radiol In press

4. Arthurs OJ, Taylor AM, Sebire NJ (2014) Indications, advantages and limitations of perinatal post mortem imaging in clinical practice. Pediatr Radiol http://dx.doi.org/10.1007/s00247-014-3165-z

5. Breeze AC, Jessop FA, Set PA et al (2011) Minimally-invasive fetal autopsy using magnetic resonance imaging and percutaneous organ biopsies: clinical value and comparison to conventional autopsy. Ultrasound Obstet Gynecol 37:317-323

6. Thayyil S, Sebire NJ, Chitty LS, MARIAS collaborative group et al (2013) Post-mortem MRI versus conventional autopsy in fetuses and children: a prospective validation study. Lancet 382: 223-233

7. Thayyil S, De Vita E, Sebire NJ et al (2012) Post-mortem cerebral magnetic resonance imaging $\mathrm{T} 1$ and $\mathrm{T} 2$ in fetuses, newborns and infants. Eur J Radiol 81:e232-e238
8. Humphries PD, Sebire NJ, Siegel MJ, Olsen ØE (2007) Tumors in pediatric patients at diffusion-weighted MR imaging: apparent diffusion coefficient and tumor cellularity. Radiology 245:848-854

9. McDonald K, Sebire NJ, Anderson J, Olsen OE (2011) Patterns of shift in ADC distributions in abdominal tumours during chemotherapy-feasibility study. Pediatr Radiol 41:99-106

10. Gawande RS, Gonzalez G, Messing S, Khurana A, Daldrup-Link HE (2013) Role of diffusion-weighted imaging in differentiating benign and malignant pediatric abdominal tumors. Pediatr Radiol 43:836-845

11. Clark MA, Worrell MB, Pless JE (1996) Post mortem changes in soft tissues. In: Haglung WD, Sorg MH (eds). Forensic taphonomy: the postmortem fate of human remains, 1st edn. CRC Press, Boca Raton. Chapter 9; 151-164

12. Seehaus AK, Roebroeck A, Chiry O et al (2013) Histological validation of DW-MRI tractography in human postmortem tissue. Cereb Cortex 23:442-450

13. Sun X, Wang H, Chen F et al (2009) Diffusion-weighted MRI of hepatic tumor in rats: comparison between in vivo and postmortem imaging acquisitions. J Magn Reson Imaging 29:621-628

14. D'Arceuil H, de Crespigny A (2007) The effects of brain tissue decomposition on diffusion tensor imaging and tractography. Neuroimage 36:64-68

15. Shepherd TM, Flint JJ, Thelwall PE et al (2009) Postmortem interval alters the water relaxation and diffusion properties of rat nervous tissue-implications for MRI studies of human autopsy samples. Neuroimage 44:820-826

16. Kim JH, Trinkaus K, Ozcan A, Budde MD, Song SK (2007) Postmortem delay does not change regional diffusion anisotropy characteristics in mouse spinal cord white matter. NMR Biomed 20:352-359

17. Scheurer E, Lovblad KO, Kreis R et al (2011) Forensic application of postmortem diffusion-weighted and diffusion tensor MR imaging of the human brain in situ. AJNR Am J Neuroradiol 32:1518-1524

18. Thayyil S, Sebire NJ, Chitty LS et al (2011) Post mortem magnetic resonance imaging in the fetus, infant and child: A comparative study with conventional autopsy (MaRIAS protocol). BMC Pediatr 11:120

19. Kozak LR, Bango M, Szabo M, Rudas G, Vidnyanszky Z, Nagy Z (2010) Using diffusion MRI for measuring the temperature of cerebrospinal fluid within the lateral ventricles. Acta Paediatr 99:237-243

20. Jones RA, Grattan-Smith JD (2003) Age dependence of the renal apparent diffusion coefficient in children. Pediatr Radiol 33:850-854

21. Hagmann P, Jonasson L, Maeder P, Thiran JP, Wedeen VJ, Meuli R (2006) Understanding diffusion MR imaging techniques: from scalar diffusion-weighted imaging to diffusion tensor imaging and beyond. Radiographics 26:S205-S223

22. Le Bihan D (2013) Apparent Diffusion Co-efficient and Beyond: What diffusion MR imaging can tell us about Tissue structure. Radiology 268:318-322

23. Savelli S, Di Maurizio M, Perrone A, Tesei J, Francioso A, Angeletti $\mathrm{M}$ et al (2007) MRI with diffusion-weighted imaging (DWI) and apparent diffusion coefficient (ADC) assessment in the evaluation of normal and abnormal fetal kidneys: preliminary experience. Prenat Diagn 27:1104-1111

24. Manganaro L, Perrone A, Sassi S, Fierro F, Savelli S, Di Maurizio M et al (2008) Diffusion-weighted MR imaging and apparent diffusion coefficient of the normal fetal lung: preliminary experience. Prenat Diagn 28:745-748

25. Balassy C, Kasprian G, Brugger PC, Csapo B, Weber M, Hörmann M et al (2008) Diffusion-weighted MR imaging of the normal fetal lung. Eur Radiol 18:700-706

26. Lee W, Krisko A, Shetty A, Yeo L, Hassan SS, Gotsch F et al (2009) Non-invasive fetal lung assessment using diffusion-weighted imaging. Ultrasound Obstet Gynecol 34:673-677 